\title{
Collective dynamics out of thermodynamic equilibrium
}

\author{
Gordon Robb ${ }^{1, *}$ and Antonio Politi ${ }^{2, \dagger}$ \\ ${ }^{1}$ Department of Physics and SUPA, University of Strathclyde, John Anderson Building, 107 Rottenrow, Glasgow G4 ONG, United Kingdom \\ ${ }^{2}$ Institute for Complex Systems and Mathematical Biology and SUPA, University of Aberdeen, Aberdeen AB24 3UE, United Kingdom
}

(Received 13 June 2016; published 3 April 2017)

\begin{abstract}
Thorough numerical studies reveal that spatially extended dissipative systems with long-range interactions may give rise to a large-scale dynamics. This phenomenon, which generalizes mean-field chaos, can be interpreted as a form of subtle pattern formation, where a chaotic microscopic dynamics coexists with a macroscopic irregular behavior, sustained by the spontaneous emergence of long-wavelength "hydrodynamic" modes. This regime can emerge only if the coupling is sufficiently long ranged, otherwise normal space-time chaos is observed. In Stuart-Landau oscillators a further regime is found, where the amplitude of the hydrodynamic modes exhibits an anomalous intermediate scaling between that of collective and standard space-time chaos.
\end{abstract}

DOI: 10.1103/PhysRevE.95.040201

Understanding the behavior of systems steadily kept out of equilibrium is one of the most formidable tasks currently confronting physicists. The presence of many different spatial and temporal scales such as those, for instance, encountered in turbulence and neuroscience is in fact one of the many different facets in which the complexity of the problem manifests itself. So far, a large amount of effort has been focused on understanding steady macroscopic states in stochastic models, as they are more amenable to analytical treatments. The asymmetric exclusion process is one of the most prominent such examples, where different phases have been detected and fully characterized (see, e.g., Refs. [1-3]). In general, however, the absence of detailed balance and the presence of long-range interactions (see, e.g., Ref. [4]) make even the development of heuristic arguments rather problematic, so that most of the investigations are inevitably of a numerical nature.

Currently, mean-field-type models are an active field of research, as this is the simplest setup where collective dynamics can be investigated. For instance, in both logistic maps [5] and Stuart-Landau oscillators [6,7], macroscopic observables have been proven to exhibit a complex dynamics $[8,9]$. The collective behavior should not be confused with the possibly chaotic microscopic evolution: It refers to average variables whose fluctuations go beyond the usual statistical fluctuations due to the finite number of elements they are composed of. Evidence of irregular evolution has been found in sparse networks [10]. However, nothing is known about such dynamics in spatially extended systems. Early simulations of nonlinear oscillators embedded in regular lattices revealed no more than (collective) periodic oscillations: This is the case of both cellular automata and coupled maps [11-13].

In this Rapid Communication we contribute to bridge this gap by investigating one-dimensional systems where the coupling of the local variable $v_{j}$ with the rest of the chain is expressed by a term proportional to

$$
\left\langle v_{j}\right\rangle_{G} \equiv \sum_{j} G(j-m) v_{m}(t)
$$

*g.r.m.robb@strath.ac.uk

†a.politi@abdn.ac.uk where $G$ expresses the coupling strength. A constant $G(n)$ corresponds to a mean-field model. Here, we focus on $G(n)=$ $K_{\delta} n^{-\delta}(n \neq 0)[14]$ and $G(n)=A+B \cos (2 \pi n / N)$.

The former coupling type is often invoked in statistical mechanics as a surrogate for the (numerically) more expensive simulations in spaces of increasing dimension. In fact, for small $\delta$, the dynamics is substantially equivalent to that of a system embedded in a high-dimensional space, while large $\delta$ values correspond to effectively short-range interactions in low-dimensional spaces. One example of such studies is the effective breaking of ergodicity in spin glasses [15-17]. The Hamiltonian dynamics of self-gravitating systems is another setup where slowly decaying interactions are naturally present [18-20]. Further examples are Bose-Einstein condensates (BECs) [21], ion chains [22], and strongly correlated condensed matter systems [23], where the role of the interaction range of a system undergoing a "quantum quench" has been an area of active recent study. Finally, setups with no collective stationary dynamics such as the Kuramoto model have been investigated to determine the critical dimension, where qualitative changes in the synchronized regimes are to be expected [24]. The sinusoidal coupling scheme is instead a key ingredient in another class of nonequilibrium collective phenomena, chimera states, where identical oscillators split into two groups with distinct synchronization properties $[25,26]$.

Standard space-time chaos, such as that arising in the Ginzburg-Landau or the Kuramoto-Sivashinsky equation [27], can be characterized in terms of the scaling behavior of the Fourier modes $\tilde{V}_{k}=(1 / N) \sum_{j} v_{j} e^{-2 \pi i k j / N}$ (where $N$ is the system size): Their amplitude is of order $1 / N$ (with the possible exception of a constant zero mode), meaning that the "energy" is distributed over all wavelengths (up to some ultraviolet cutoff). The collective chaos observed in mean-field models is instead characterized by finite temporal fluctuations of the average variable, i.e., of $\tilde{V}_{0}$.

Here, we show that a spatially structured collective chaos may arise, where the amplitude of low- $k$ Fourier modes remains finite in the thermodynamic limit. Collective chaos emerges in a finite range of $\delta$ values, suggesting that it can be observed also in regular lattices of sufficiently high dimension. At variance with hydrodynamic systems, where the relevant 
low- $k$ modes are associated with the diffusive dynamics of some conserved quantities (e.g., energy, mass, etc.), here there are no such relationships. The study of the sinusoidal coupling suggests that sufficiently long-ranged interactions may suffice to trigger low- $k$ modes.

We have studied two models: (i) logistic maps (LMs) and Stuart-Landau oscillators (SLOs). The former one offers the advantage of faster simulations and thereby the possibility to access larger system sizes; the latter one is appealing, as it corresponds to the normal form for dynamical systems which have undergone a Hopf bifurcation and is often used to test different synchronization scenarios. In SLOs, a second anomalous regime exists where the dynamics is dominated by low- $k$ modes, whose amplitude decreases slower than $1 / N$.

Coupled maps. Given the scalar variable $u_{j}(t) \in[0,1]$ defined on site $j$ at time $t$, the evolution rule is $u_{j}(t+1)=$ $v_{j}(t)+g\left[\left\langle v_{j}(t)\right\rangle_{G}-v_{j}(t)\right]$, where $v_{j}(t)=a u_{j}(t)\left[1-u_{j}(t)\right]$ is the result of a logistic transformation, while $\left\langle v_{j}\right\rangle_{G}$ is defined as in Eq. (1). The power-law coupling is conveniently estimated in Fourier space, where it reduces to the product between $\tilde{V}_{k}$ and the transform $\tilde{G}_{k}$ of $G(n)$, thereby reducing the number of operations from $N^{2}$ to $N \log N$ [28].

In the uncoupled case, the time average $\left\langle\tilde{V}_{k}\right\rangle_{t}$ of each Fourier mode is equal to zero, except for $\tilde{V}_{0}$. In order to get rid of this trivial contribution we decided to monitor the temporal standard deviation defined as $\sigma_{k}^{2}=\left\langle\left|\tilde{V}_{k}-\left\langle\tilde{V}_{k}\right\rangle_{t}\right|^{2}\right\rangle_{t}$, with an obvious meaning of the symbols.

In the mean-field limit, the onset of collective dynamics is signaled by a nonzero $\sigma_{0}$, while all other $\sigma_{k}$ 's are equal to one another and of order $1 / \sqrt{N}$, due to their statistical origin.

How does this scenario change when $\delta$ is increased from zero? The results for typical parameter values are presented in Fig. 1. There, we can see that the collective dynamics persists up to the critical point $\delta_{c} \approx 1.15$, beyond which $\sigma_{0}$ is no longer finite, decreasing as $1 / \sqrt{N}$ with the system size.

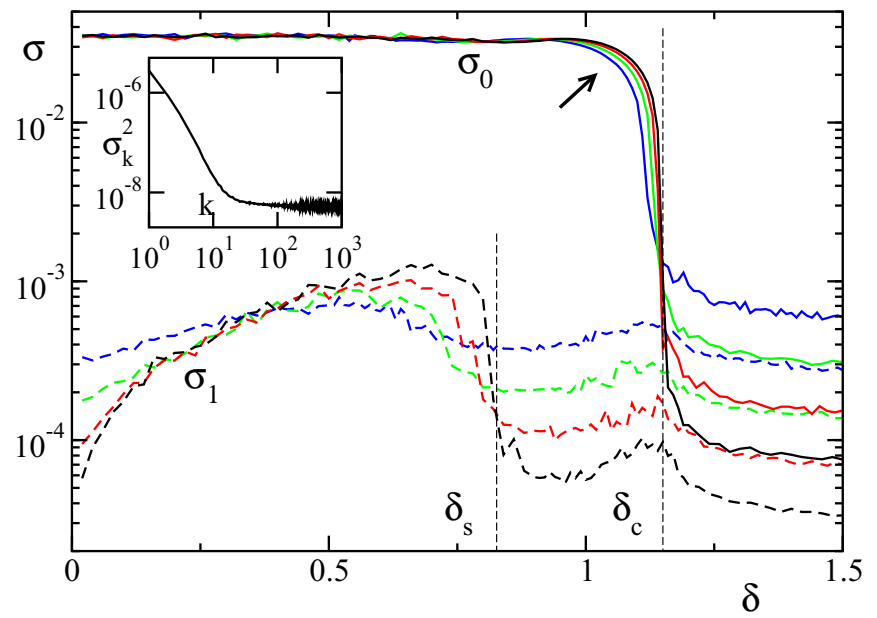

FIG. 1. The standard deviation of the first two Fourier modes in a chain of coupled logistic maps with $a=3.9$ and $g=0.1$. The solid (dashed) curves refer to standard deviation of the zeroth (first) mode. Blue, green, red, and black lines correspond to $N=2^{17}, 2^{19}$, $2^{21}$, and $2^{23}$, respectively. The two vertical dashed lines identify two transitions (see the text). In the inset, the variance $\sigma_{k}^{2}$ is plotted vs $k$ for $N=2^{23}$ and $\delta=0.7$.
Given the tendency of $\delta_{c}$ to shift to the right for increasing $N$, it follows that its asymptotic value is convincingly larger than 1 , i.e., long-range dynamical order is supported beyond the point where the sum of the interaction terms diverges. Furthermore, the tendency to shift rightwards suggests that the transition is discontinuous. No evidence of hysteretical behavior has been found while sweeping the parameter $\delta$ back and forth.

The amplitude of $\sigma_{1}$ provides information on the spatial structure: a nonexisting feature of mean-field models. Above $\delta_{s} \approx 0.82$, all $\sigma_{k}$ for $k>0$ scale as $1 / \sqrt{N}$, implying that they behave as in standard space-time chaos. Below $\delta_{s}, \sigma_{1}$ remains finite, being possibly proportional to $\delta$ for $\delta \ll 1$. The existence of a spatial structure is not only due to a finite zeroth Fourier mode: An entire range of "hydrodynamic" modes is indeed present. An instance can be seen in the inset of Fig. 1, where the low- $k$ spectrum is reported for $\delta=0.7$ and $N=2^{23}$. There, one can appreciate an approximate power-law decay of the Fourier modes, $\sigma_{k}^{2} \approx k^{-2}$. All in all, the fraction of power contained in the low- $k$ modes is about $38 \%$. The same scenario is found in a finite range of $\delta$ values.

In order to establish the complexity of the collective dynamics, we start looking at the return map of $V_{0}(t)$. In Fig. 2 results are reported for two $\delta$ values (for two different system sizes). For $\delta=0.7$ [Fig. 2(a)], the points fill a blurred area which is essentially unchanged when the system size is increased. This is analogous to what is observed in the meanfield limit [8]. For $\delta=1$ [Fig. 2(b)], a more regular dynamical behavior is observed. The decreasing thickness suggests that it could be quasiperiodic in the thermodynamic limit.

A more quantitative analysis can be performed by embedding $V_{0}(t)$ in spaces of increasing dimension $d_{E}$ and thereby estimating the effective fractal dimension $D_{E}(\varepsilon)$ as a function of the resolution $\varepsilon$. We have implemented a fixed-mass method
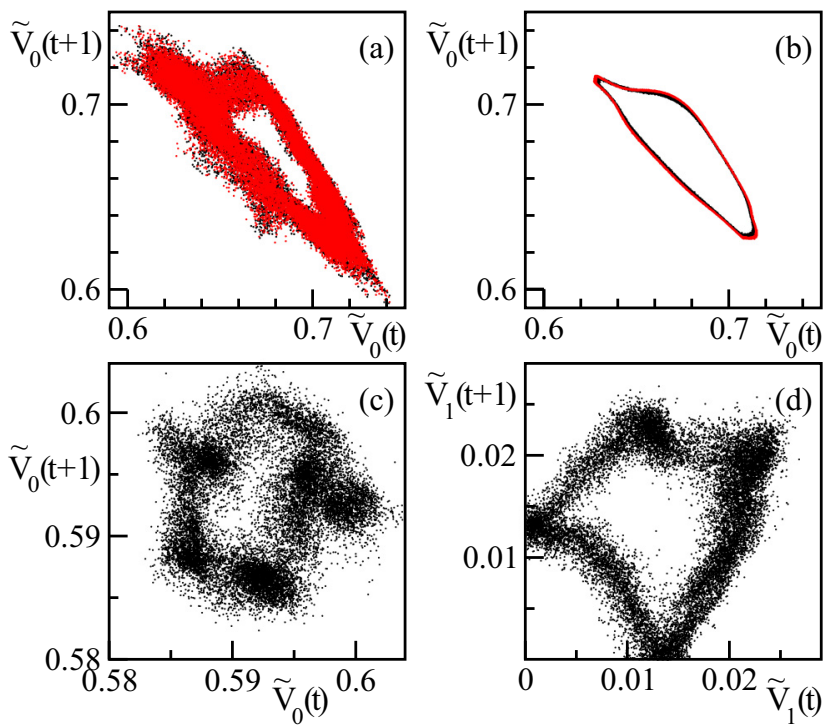

FIG. 2. Return map for the amplitude of the zeroth and first Fourier modes for different setups involving logistic maps. The first two panels refer to power-law coupling for (a) $\delta=0.7$ and (b) $\delta=1$.0. In both cases, black dots correspond to $N=2^{19}$, while red dots correspond to $N=2^{21}$. The last two panels refer to a purely sinusoidal coupling for $N=2^{22}$. 

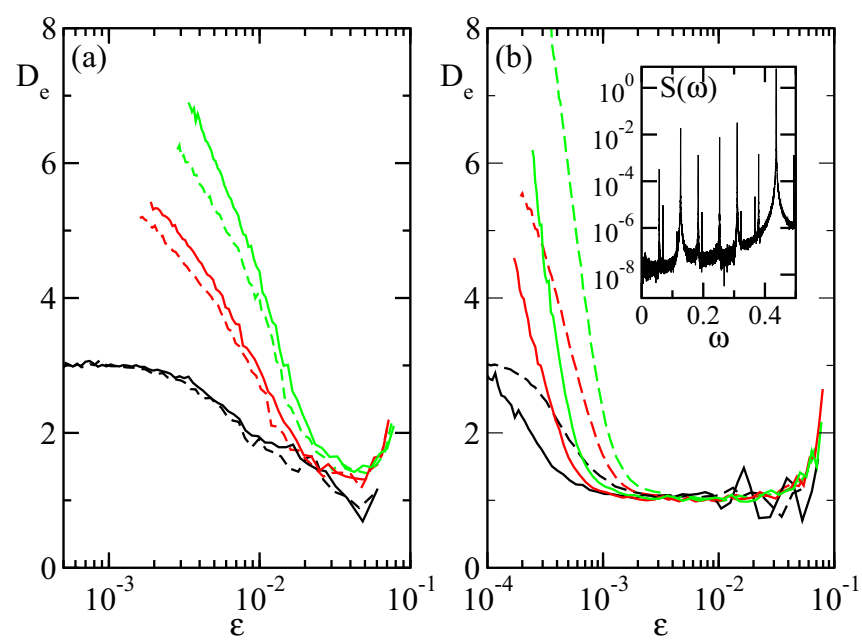

FIG. 3. Fractal dimension of the zeroth Fourier mode, once embedded in spaces of increasing dimension (black, red, and green colors correspond to embedding dimension $d_{E}=3,5$, and 12 , respectively). Dashed lines correspond to $N=2^{19}$, and solid lines to $N=2^{21}$. (a) and (b) correspond to $\delta=0.7$ and $\delta=1$, respectively. The power spectrum in the inset has been obtained for $N=2^{21}$.

[29], as it has the advantage of self-selecting the range of statistically meaningful $\varepsilon$ distances. The method requires comparing $N_{r}$ reference points with an increasing number $m$ of measurement points to thereby determine the distance $d_{p}$ of the $p$ th nearest neighbor. The dimension $D_{E}(\varepsilon)$ would then be obtained from the scaling behavior of the average $\varepsilon$ of $d_{p}$ (see Ref. [30] for further details). In Fig. 3(a) we see that for $\delta=0.7, D_{e}$ steadily increases for $\varepsilon \rightarrow 0$ with a reasonable evidence of a convergence towards $d_{E}$ itself (for the smaller $d_{E}$ values). The lack of an appreciable dependence on the system size $N$ reveals that the resolution dependence of the dimension is not due to microscopic fluctuations.

This result can be better appreciated by looking at Fig. 3(b), where the results for $\delta=1$ are reported. There, we see that upon increasing $N$, the increase of $D_{E}$ is "delayed" to smaller scales, suggesting that the fluctuations seen in Fig. 2 are nothing but a manifestation of finite-size fluctuations around a one-dimensional attractor. This interpretation is partially confirmed by the structure of the power spectrum of $V_{0}(n)$, which is composed of several narrow lines (see the inset in Fig. 3). The dynamical regime is, nevertheless, more complex than one could expect, since all spectral lines are multiples of two distinct frequencies (the first two peaks). This suggests that two degrees of freedom are active, although one amplitude is pretty small and thus difficult to detect with a fractal-dimension analysis. Nevertheless, the dynamical complexity decreases while increasing $\delta$, until, above $\delta_{c}$, no collective motion can be sustained at all.

In order to shed some light on how finite "low- $k$ " modes can be self-sustained, we investigated the sinusoidal coupling scheme $G(n)=A+B \cos (2 \pi n / N)$. This scheme corresponds to truncating $\tilde{G}_{k}$ to include only the zeroth and first Fourier modes. In other words, this is the simplest scheme which goes beyond the standard mean field. Actually, it is also the scheme often used to analyze chimera states [26]. In order

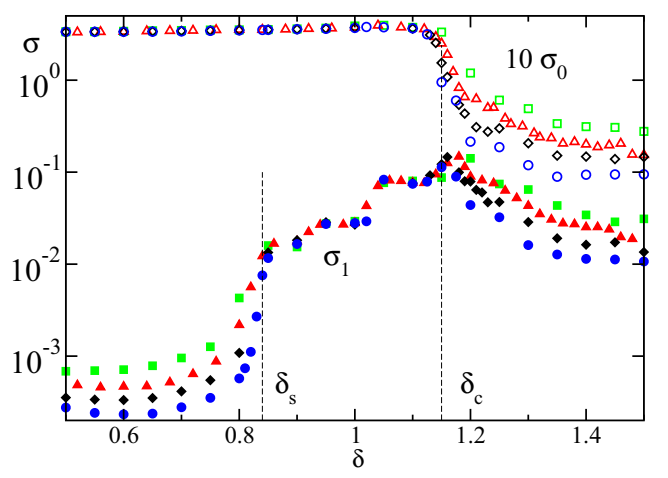

FIG. 4. The fluctuations of the first two Fourier modes for a chain of Stuart-Landau oscillators. Green, red, black, and blue symbols correspond to $N=2^{14}, 2^{15}, 2^{16}$, and $2^{17}$ oscillators, respectively (open and solid symbols correspond to the zeroth and first mode, respectively). For the sake of clarity, the data for $\sigma_{0}$ have been multiplied by a factor 10 . The two dashed vertical lines identify two transitions.

to appreciate the potential role of the nonzero harmonics, we have set $A=0$ and $B=1$, i.e., we have studied the model $u_{j}(t+1)=v_{j}(t)+(g / N) \sum_{m} \cos \left[\frac{2 \pi}{N}(j-m) / N\right]$, with $g=$ 0.1 as before, so that the standard mean-field coupling is absent. As shown in Figs. 2(c) and 2(d), a sinusoidal coupling function can alone yield a collective chaos. Even more interesting, the presence of the first harmonic alone is able to induce a nontrivial dynamics of the zeroth mode.

Stuart-Landau oscillators. Next, we have investigated a chain of SLOs, whose evolution is ruled by the equation

$\dot{v}_{j}=v_{j}-\left(1+i c_{2}\right)\left|v_{j}\right|^{2} v_{j}+k\left(1+i c_{1}\right)\left[\left\langle v_{j}(t)\right\rangle_{G}-v_{j}(t)\right]$,

where now the variable $v_{j}$ is complex. Most of the simulations have been performed by referring to the parameter values selected in Ref. [6], $c_{1}=-2.5, c_{2}=3, k=0.445$. One can again use the trick of determining the velocity field in Fourier space, thus reducing the computational complexity. The resulting scenario is summarized in Fig. 4.

As in LMs, a finite zeroth mode survives up until $\delta \approx$ 1.15. However, here the additional Fourier modes exhibit a drastically different dependence on $\delta$. They are negligible up until a critical value $\delta_{s} \approx 0.8$, implying that the mean-field scenario remains valid in a finite range of $\delta$ values. Above $\delta_{s}$, the "hydrodynamic" modes are self-sustained and even of the same size as the zeroth mode. As a consequence, the collapse of the collective dynamics above $\delta_{c}$ here follows a different scenario. First of all, the disappearance of macroscopic features seems not to be discontinuous (this is confirmed by preliminary simulations performed for $c_{1}=-2, c_{2}=3$, $k=0.47$, the set of parameters selected in Ref. [9]): The process is a sort of intermittency, where patches of collective dynamics are interspersed on a background of a less coherent regime.

The analysis of the hydrodynamic component reveals, nevertheless, a strong similarity with the previous case. The spectra reported in Fig. 5(a) (obtained for $\delta=1.1$ ) exhibit again a power-law decay with a similar exponent, $\sigma_{k}^{2} \approx k^{-1.7}$. 

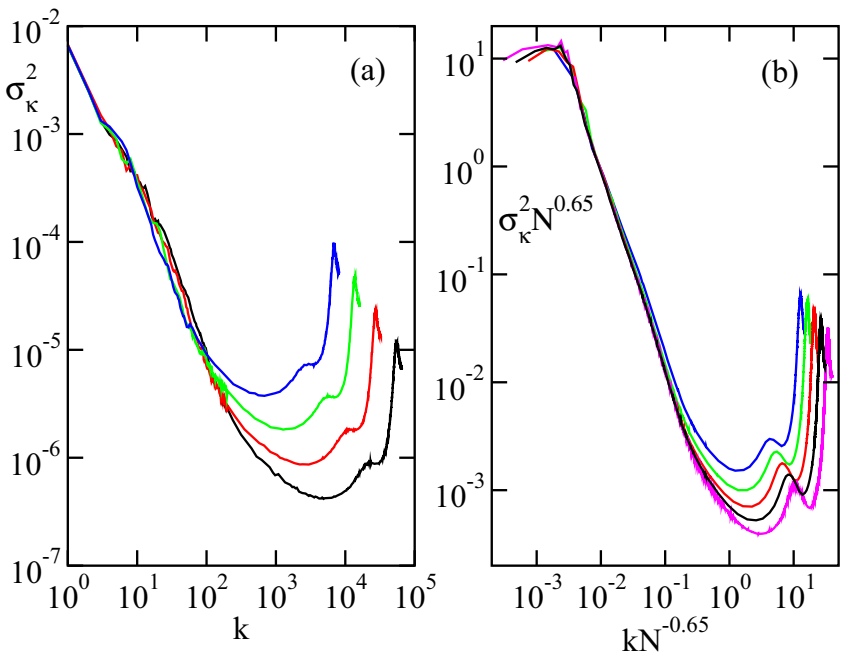

FIG. 5. The (spatial) power spectrum for a chain of SL oscillators for (a) $\delta=1.1$ and (b) $\delta=1.2$. The color code is the same in both cases: Magenta, black, red, green, and blue lines correspond to $N=$ $2^{17}, 2^{16}, 2^{15}, 2^{14}$, and $2^{13}$.

Also in this case, the collective dynamics survives above $\delta=1$. Before the collapse, the most relevant contribution is given by finite- $k$ modes. The different scenario finally includes an anomalous scaling of the Fourier modes, as shown in Fig. 5(b), which refers to $\delta=1.2$, where the evolution is neither collective (no finite modes exist), nor spatially extended in the usual sense (with a $1 / N$ scaling of all square amplitudes). Our simulations in fact give evidence of an exponent of - 0.65 [31].

As for LMs, we have performed a time-series analysis of the leading modes for different $\delta$ values. Here, the collective dynamics is everywhere high dimensional, including the crossover region where $\delta \approx 1$. We attribute this to the fact that each Stuart-Landau oscillator has an additional degree of freedom (the rotation), which is marginally stable in the uncoupled limit and therefore very responsive to coupling fluctuations. In fact, higher-order Fourier modes are active as well. Finally, we have verified that a collective spatially orga- nized structure emerges also when a chimeralike sinuosoidal coupling is introduced (data not shown).

Discussion and conclusions. Mutual coupling of nonlinear dynamical systems can drastically change the behavior of the single units. In the literature, such variations are sometimes referred to as manifestations of a collective dynamics. A much subtler manifestation is the onset of macroscopic phases. In this Rapid Communication we have shown that collective dynamics can emerge in spatially extended dissipative dynamical systems in spite of the "decorrelation" induced by microscopic chaos. Both in discrete- and continuous-time models, sinusoidal as well as power-law-type interactions can induce a spatially structured macroscopic dynamics, which goes beyond the global averages of mean-field setups. This is a nontrivial form of pattern formation, nontrivial since it cannot be inferred from a stability analysis of the microscopic equations. In the context of the SLO setup, it can be seen as a generalized chimera state: a spatial structure spontaneously formed in a system of identical oscillators. In contrast to standard chimeras, here the spatial structure fluctuates irregularly. In fact, a fractal-dimension analysis reveals that the collective motion is typically high dimensional. It would be interesting to understand to what extent it can be described in terms of a few nonlinear stochastic equations, as it happens in fluctuating hydrodynamics. Nevertheless, the analogy with hydrodynamics should not be pushed too far, as the time scales do not grow with the system size. The emergence of collective chaos for the simple sinusoidal coupling suggests the possibility to develop an analytical treatment in Fourier space.

In both models, the collective dynamics is sustained only below a critical value $\delta_{c}$. From a statistical-mechanics viewpoint, this phenomenon can be interpreted as the existence of a critical dimension below which large-scale dynamics cannot be sustained. Whether the closeness between the actual values of $\delta_{c}$ found in the LM and SLO models is an indication of universality is too early to conclude. Additional simulations as well as theoretical arguments are needed.

Some results were obtained using the EPSRC funded ARCHIE-WeSt High Performance Computer (www.archiewest.ac.uk). EPSRC under Grant No. EP/K000586/1.
[1] F. Spitzer, Adv. Math. 5, 246 (1970).

[2] B. Derrida, M. R. Evans, V. Hakim, and V. Pasquier, J. Phys. A: Math. Gen. 26, 1493 (1993).

[3] T. Chou, K. Mallick, and R. K. P. Zia, Rep. Prog. Phys. 74, 116601 (2011).

[4] L. Bertini, A. De Sole, D. Gabrielli, G. Jona-Lasinio, and C. Landim, J. Stat. Phys. 135, 857 (2009).

[5] K. Kaneko, Phys. Rev. Lett. 65, 1391 (1990).

[6] N. Nakagawa and Y. Kuramoto, Prog. Theor. Phys. 89, 313 (1993); Physica D 80, 307 (1995).

[7] V. Hakim and W. J. Rappel, Phys. Rev. A 46, R7347 (1992).

[8] T. Shibata, T. Chawanya, and K. Kaneko, Phys. Rev. Lett. 82, 4424 (1999).

[9] K. A. Takeuchi and H. Chaté, J. Phys. A 46, 254007 (2013).
[10] S. Luccioli, S. Olmi, A. Politi, and A. Torcini, Phys. Rev. Lett. 109, 138103 (2012).

[11] H. Chaté and P. Manneville, Europhys. Lett. 14, 409 (1991).

[12] J. A. C. Gallas, P. Grassberger, H. J. Herrmann, and P. Ueberholz, Physica A 180, 19 (1992).

[13] H. Chaté and P. Manneville, Prog. Theor. Phys. 87, 1 (1992).

[14] The constant $K_{\delta}$ is determined by imposing that the total sum is equal to 1 . Moreover, the distance $|j-m|$ is computed taking into account the periodic boundary conditions.

[15] H. G. Katzgraber and A. P. Young, Phys. Rev. B 67, 134410 (2003).

[16] H. G. Katzgraber, A. K. Hartmann, and A. P. Young, Phys. Proc. 6, 35 (2010).

[17] C. Monthus, J. Stat. Mech. (2014) P06015. 
[18] A. Campa, T. Dauxois, and S. Ruffo, Phys. Rep. 480, 57 (2009).

[19] Sh. Gupta, M. Potters, and S. Ruffo, Phys. Rev. E 85, 066201 (2012).

[20] M. Joyce, J. Morand, F. Sicard, and P. Viot, Phys. Rev. Lett. 112, 070602 (2014).

[21] D. O’Dell, S. Giovanazzi, G. Kurizki, and V. M. Akulin, Phys. Rev. Lett. 84, 5687 (2000).

[22] J. Schachenmayer, B. P. Lanyon, C. F. Roos, and A. J. Daley, Phys. Rev. X 3, 031015 (2013).

[23] Y. Wang, B. Moritz, C.-C. Chen, C. J. Jia, M. van Veenendaal, and T. P. Devereaux, Phys. Rev. Lett. 116, 086401 (2016).

[24] Sh. Gupta, A. Campa, and S. Ruffo, J. Stat. Mech. (2014) R08001.
[25] Y. Kuramoto and D. Battogtokh, Nonlinear Phenom. Complex Syst. 5, 380 (2002).

[26] D. M. Abrams and S. H. Strogatz, Int. J. Bifurcation Chaos Appl. Sci. Eng. 16, 21 (2006).

[27] H. G. Schuster and W. Just, Spatiotemporal Chaos, in Deterministic Chaos: An Introduction, 4th ed. (Wiley-VCH, Weinheim, 2005).

[28] M. Cencini and A. Torcini, Physica D 208, 191 (2005).

[29] R. Badii and A. Politi, J. Stat. Phys. 40, 725 (1985).

[30] E. Ullner and A. Politi, Phys. Rev. X 6, 011015 (2016).

[31] An anomalous scaling of the zero mode was previously observed in in a mean-field setup: See H. Hong, H. Chaté, K.-H. Tang, and H. Park, Phys. Rev. E 92, 022122 (2015). 